\title{
$\beta$-lactam antibiotic concentrations during continuous renal replacement therapy
}

\author{
Marjorie Beumier ${ }^{1}$, Giuseppe Stefano Casu ${ }^{1}$, Maya Hites ${ }^{2}$, Lucie Seylerr ${ }^{2}$, Frederic Cotton ${ }^{3}$, Jean-Louis Vincent ${ }^{1}$, \\ Frédérique Jacobs ${ }^{2}$ and Fabio Silvio Taccone ${ }^{1 *}$
}

\begin{abstract}
Introduction: The use of standard doses of $\beta$-lactam antibiotics during continuous renal replacement therapy (CRRT) may result in inadequate serum concentrations. The aim of this study was to evaluate the adequacy of unadjusted drug regimens (i.e., similar to those used in patients with normal renal function) in patients treated with CRRT and the influence of CRRT intensity on drug clearance.

Methods: We reviewed data from 50 consecutive adult patients admitted to our Department of Intensive Care in whom routine therapeutic drug monitoring (TDM) of broad-spectrum $\beta$-lactam antibiotics (ceftazidime or cefepime, CEF; piperacillin/tazobactam; TZP; meropenem, MEM) was performed using unadjusted $\beta$-lactam antibiotics regimens $(C E F=2 \mathrm{~g}$ q8h; TZP = $4 \mathrm{~g}$ q6h; MEM = $1 \mathrm{~g}$ q8h). Serum drug concentrations were measured twice during the elimination phase by high-performance liquid chromatography (HPLC-UV). We considered therapy was adequate when serum drug concentrations were between 4 and 8 times the minimal inhibitory concentration (MIC) of Pseudomonas aeruginosa during optimal periods of time for each drug ( $\geq 70 \%$ for CEF; $\geq 50 \%$ for TZP; $\geq 40 \%$ for MEM). Therapy was considered as early (ET) or late (LT) phase if TDM was performed within 48 hours of antibiotic initiation or later on, respectively.
\end{abstract}

Results: We collected 73 serum samples from 50 patients (age $58 \pm 13$ years; Acute Physiology and Chronic Health Evaluation II (APACHE II) score on admission 21 (17-25)), 35 during ET and 38 during LT. Drug concentrations were above 4 times the MIC in 63 (90\%), but above 8 times the MIC in 39 (53\%) samples. The proportions of patients with adequate drug concentrations during ET and LT were quite similar. We found a weak but significant correlation between $\beta$-lactam antibiotics clearance and CRRT intensity.

Conclusions: In septic patients undergoing CRRT, doses of $\beta$-lactam antibiotics similar to those given to patients with normal renal function achieved drug levels above the target threshold in 90\% of samples. Nevertheless, 53\% of samples were associated with very high drug levels and daily drug regimens may need to be adapted accordingly.

\section{Introduction}

Sepsis is a major cause of morbidity and mortality in critically ill patients [1,2]. Early and adequate antibiotic therapy in this population is crucial to maximize chances of survival [3-6]. $\beta$-lactam antibiotics are widely used as first-line therapy in septic patients and are particularly effective against bacteria less susceptible to other antibiotics, such as Pseudomonas aeruginosa. These drugs have a time-dependent antibacterial activity and the best pharmacodynamic parameter to predict their efficacy is

\footnotetext{
* Correspondence: ftaccone@ulb.ac.be

'Department of Intensive Care, Erasme Hospital, Université Libre de Bruxelles (ULB), Route de Lennik, 808, 1070 Brussels, Belgium

Full list of author information is available at the end of the article
}

the time during which their serum concentration is above the minimal inhibitory concentration (MIC) of the pathogen [7].

Sepsis may significantly alter antibiotic pharmacokinetics (PK). In particular, volume of distribution ( $\mathrm{Vd}$ ) may increase because of fluid resuscitation and capillary leakage, whereas increased cardiac output may promote augmented renal clearance and drug elimination [7]. Thus, insufficient antibiotic concentrations may occur in septic patients receiving standard antibiotic doses, potentially leading to therapeutic failure and encouraging development of antimicrobial resistance $[7,8]$. However, antibiotic PK can vary considerably during critical illness; for example, acute kidney injury (AKI), which commonly

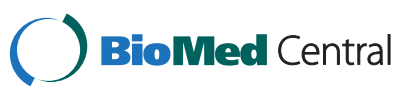


complicates sepsis, alters antibiotic elimination, leading to drug accumulation [9]. The use of continuous renal replacement therapy (CRRT) may further alter antibiotic PK. Nevertheless, current recommendations on antibiotic dosing during CRRT [10] are based on studies that included a limited number of patients, with varying inclusion/exclusion criteria and who received different types of RRT [11-13]. Indeed, Roberts et al. showed a great variability in $\beta$-lactam antibiotics concentrations in critically ill patients treated with CRRT [14]. Moreover, in a prospective study, Seyler et al. showed that the recommended doses for broad-spectrum $\beta$-lactam antibiotics were largely insufficient to maintain therapeutic serum concentrations for the treatment of $P$. aeruginosa in septic patients [15]. The authors suggested the use of $\beta$-lactam antibiotics doses similar to those used in patients without renal failure, at least during the first days of treatment in this population. The aim of the present study was, therefore, to evaluate the adequacy of this dosage strategy in septic patients treated with CRRT and to evaluate the influence of CRRT intensity on drug clearance.

\section{Patients and methods}

\section{Study design and inclusion criteria}

Since December 2009, patients undergoing CRRT in our unit, who require treatment with $\beta$-lactam antibiotics receive doses similar to those used in patients with normal renal function. We therefore reviewed data from all adult patients admitted to the 35-bed Department of Intensive Care of Erasme University Hospital, Brussels between January 2010 and November 2011. Inclusion criteria were: a) diagnosis of severe sepsis or septic shock according to standard criteria [16]; b) therapy with broad-spectrum $\beta$-lactam antibiotics (ceftazidime or cefepime (CEF), piperacillin/tazobactam (TZP) or meropenem $(\mathrm{MEM}))$, given at the usual doses $(\mathrm{CEF}=2 \mathrm{~g}$ $\mathrm{q} 8 \mathrm{~h} ; \mathrm{TZP}=4 \mathrm{~g}$ q6h; MEM $=1 \mathrm{~g}$ q8h); c) AKI treated with CRRT; d) residual creatinine clearance $(\mathrm{CrCl})<30 \mathrm{~mL} / \mathrm{mi}-$ nute; e) at least one therapeutic drug monitoring (TDM) sample taken during the CRRT treatment. Exclusion criteria were burns, cystic fibrosis and the use of extracorporeal membrane oxygenation (ECMO) therapy. The Ethics Committee of Erasme Hospital approved the study protocol waiving the need for informed consent in view of its retrospective nature.

\section{Indications for therapeutic drug monitoring}

The choice of antibiotic was made by the attending physician. Since October 2009, TDM of broad-spectrum $\beta$-lactam antibiotics has been routinely performed in our department, especially in patients with severe sepsis or septic shock, overweight patients, patients with multidrugresistant strains of infection, transplanted patients or patients receiving extracorporeal therapies. All patients undergoing TDM are included in a prospective database, in which drug regimens, date and hours of drug sampling are recorded for each sample.

\section{Data collection}

We recorded the demographics, comorbidities, admission diagnosis, biological and microbiological (site of infection and pathogens), length of ICU and hospital stay and overall mortality for all patients. The severity of illness was assessed by the acute physiology and chronic health evaluation (APACHE) II score [17] on admission and the sequential organ failure assessment (SOFA) score [18] was recorded on the day of TDM. Treatment with vasopressors or mechanical ventilation was also recorded. Characteristics of CRRT, including dialysate rate, ultrafiltrate rate, and blood flow were recorded. CRRT intensity $(\mathrm{mL} / \mathrm{kg} / \mathrm{h})$ was calculated using the following formula:

$($ Dialysate rate $(\mathrm{mL} / \mathrm{h})+$ Ultrafiltrate $(\mathrm{mL} / \mathrm{h})) /$ Weight $(\mathrm{kg})$.

Residual creatinine clearance $(\mathrm{CrCl}, \mathrm{mL} /$ minute) was calculated from urine using the formula:

(Urine output, $\mathrm{mL}) *($ Urinary creatinine concentration, $\mathrm{mg} / \mathrm{dL}$ ) /(Serum creatinine concentration, $\mathrm{mg} / \mathrm{dL}$ ) $*$ (Time of urine collection, minutes).

\section{Pharmacokinetics analyses}

$\beta$-lactam antibiotics concentrations were determined on two blood samples $(3 \mathrm{~mL})$ drawn during the antibiotic elimination phase: one $2 \mathrm{~h}$ (T2) after a 30-minute drug infusion and the other just before the next dose administration (T0). The drug was administered over a 30-minute period by using an infusion pump, and the tubing was flushed with $0.9 \%$ sodium chloride after the dose was administered. Nurses noted the exact sampling time in the ICU patient data monitoring system (PDMS, Picis Critical Care Manager, Picis Inc., Wakefield, USA). Samples were immediately put on ice and sent to the clinical chemistry laboratory, where they were centrifuged at $3000 \mathrm{rpm}$ at $4^{\circ} \mathrm{C}$ for 10 minutes; the supernatant was then removed and analyzed using high-performance liquid chromatography connected to UV spectro-photometry (HPLC-UV), as previously reported [19]. For TZP, only piperacillin levels were measured. A one-compartment model was chosen to perform PK analyses and, assuming a reached steady-state, T0 and T2 concentrations were used to determine $\mathrm{Vd}$, total drug clearance $(\mathrm{CL})$ and elimination half-life $\left(t_{1 / 2}\right)$ [15]. Importantly, TDM results were available to clinicians but dose adjustments were performed only after a multidisciplinary discussion. Early (ET) and late (LT) phases of therapy corresponded to drug levels assessed (TDM) within $48 \mathrm{~h}$ of start of antibiotics or later on, respectively. 


\section{Clinical breakpoints and adequacy of therapy}

Adequacy of therapy was defined as drug concentrations reaching the minimal target of four times the MIC of $P$. aeruginosa; this parameter was expressed as the time in hours above four times the target MIC ( $\mathrm{T}>4 \times \mathrm{tMIC}$ ) or the percentage of time above four times the target MIC (\% T $>4 \times \mathrm{tMIC}$ ). We used the clinical breakpoints for $P$. aeruginosa as defined by the European Committee on Antimicrobial Susceptibility Testing (EUCAST): $8 \mu \mathrm{g} / \mathrm{mL}$ for CEF, $16 \mu \mathrm{g} / \mathrm{mL}$ for TZP and $2 \mu \mathrm{g} / \mathrm{mL}$ for MEM [20]. Thus, minimal target concentrations were $32 \mu \mathrm{g} / \mathrm{mL}$, $64 \mu \mathrm{g} / \mathrm{mL}$ and $8 \mu \mathrm{g} / \mathrm{mL}$ for CEF, TZP and MEM, respectively. Because of specific drug properties, such as the post-antibiotic effect or post-antibiotic leukocyte enhancement effects, the optimal\% $\mathrm{T}>4 \times \mathrm{tMIC}$ may differ between antibiotics: optimal periods of time were defined as $\geq 40 \%, \geq 70 \%$ and $\geq 50 \%$ of the dosing interval for MEM, CEF and TPZ, respectively [15]. Under-dosing was thus defined as drug levels below minimal target concentrations for optimal periods of time. Excessive drug concentrations were arbitrarily defined as those exceeding eight times the target MIC for optimal periods of time. Finally, we calculated the proportion of TDMs with insufficient $\% \mathrm{~T}>4 \times$ MIC for different MIC values.

\section{Continuous renal replacement therapy}

CRRT was initiated according to local practice. The main indications for CRRT were: metabolic acidosis $(\mathrm{pH}<7.2)$; electrolyte disturbances (especially potassium levels exceeding $6 \mathrm{mEq} / \mathrm{L}$ ); drug intoxication; fluid overload (that is, pulmonary edema) and blood urea levels $>200 \mathrm{mg} / \mathrm{dL}$. In our department CRRT is the standard of care, and standard hemodialysis or sustained low-efficiency dialysis are used only for hemodynamically stable patients before discharge to the floor. CRRT is performed through a double-lumen catheter inserted into the subclavian, femoral or internal jugular vein; continuous veno-venous hemodiafiltration (CVVHDF) or hemofiltration (CVVHF) were performed using standard equipment (Prisma or PrismaFlex, Gambro Hospal, Bologna, Italy), with a polyacrilonitrile cylinder (AN69 - Hospal, Meyzieu, France) or polysulfone (PS) hemofilters (Gambro Lundia AB, Lund, Sweden). Anticoagulation was obtained using either a systemic heparin infusion or a citrate infusion within the CRRT circuit. Initial blood flow was set at around 130 to $150 \mathrm{~mL} /$ minute and the ultrafiltrate rate was adjusted to provide at least 15 to $20 \mathrm{~mL} / \mathrm{kg} / \mathrm{h}$. Dialysate was generally used during the first 24 to $48 \mathrm{~h}$ of therapy.

\section{Statistical analysis}

Statistical analyses were performed using the SPSS 18.0 for Windows NT software package (SPSS Inc. 2004, Chicago, IL, USA). Descriptive statistics were performed for all study variables. Discrete variables are expressed as counts (percentage), and continuous variables as mean \pm SD or median (25th to 75 th percentiles). The Student $t$-test was used to assess differences between groups (early versus late). A $P$-value $<0.05$ was considered statistically significant.

\section{Results}

Fifty patients met the inclusion criteria during the study period; drug regimens remained unchanged over the study period. A total of 73 TDMs were analyzed, 35 performed during ET and 38 during LT. ICU and hospital mortality rates were $50 \%$ and $60 \%$, respectively. The median APACHE II score was 21 on admission (Table 1). Sepsis was mainly due to Gram-negative bacteria (GNB) (73\%), including $P$. aeruginosa in 18 patients. The lung was the most frequent site of infection $(n=23)$ (Table 2). At the moment the study drug was initiated in each patient, CRRT had been ongoing for 2 (0 to 3 ) days.

Thirty-two patients (64\%) were treated with MEM, 16 (32\%) with TPZ and 7 (14\%) with CEF, with 5 patients receiving two different study drugs over the CRRT period. Drug CL was significantly lower in the LT phase compared to the ET phase for patients receiving CEF, despite similar CRRT intensity and SOFA score; this

Table 1 Characteristics of patients on ICU admission and ICU outcomes

\begin{tabular}{|c|c|}
\hline & Patients $(n=50)$ \\
\hline Age, years & 59 (51 to 67$)$ \\
\hline Men/women, n & $33 / 17$ \\
\hline Body weight, kg & 75 (68 to 85$)$ \\
\hline Body mass index, $\mathrm{kg} / \mathrm{m}^{2}$ & 26.3 (23.9 to 27.9$)$ \\
\hline COPD/asthma, n (\%) & $8(16)$ \\
\hline Cardiopathy, n (\%) & $19(38)$ \\
\hline Diabetes mellitus, n (\%) & $17(34)$ \\
\hline $\mathrm{s} C \mathrm{r}>2 \mathrm{mg} / \mathrm{dL}, \mathrm{n}(\%)$ & $13(26)$ \\
\hline Liver cirrhosis, n (\%) & $18(36)$ \\
\hline Cancer, n (\%) & $7(14)$ \\
\hline Immunosuppressive agents, n (\%) & $20(40)$ \\
\hline Organ transplantation, n (\%) & $17(30)$ \\
\hline Medical admission, n (\%) & $39(78)$ \\
\hline APACHE II score on admission & 21 (17 to 25$)$ \\
\hline Lactate levels on admission, mEq/L & $2.6(1.5$ to 3.9$)$ \\
\hline Mechanical ventilation during ICU stay, n (\%) & $36(72)$ \\
\hline ICU stay, days & 15 (10 to 21$)$ \\
\hline Hospital stay, days & $23(15$ to 53$)$ \\
\hline ICU mortality, n (\%) & $25(50)$ \\
\hline Hospital mortality, n (\%) & $30(60)$ \\
\hline
\end{tabular}

Data are presented as count (percentage) or median (25th to 75 th percentiles). COPD, chronic obstructive pulmonary disease; APACHE, acute physiology and chronic health evaluation; $\mathrm{s} C r$, serum creatinine before ICU admission. 
Table 2 Characteristics of infections, identified Gramnegative pathogens and $\beta$-lactam antibioticss Number of patients $(n=50)$

\begin{tabular}{lc}
\hline Sites of infection & $23(46)$ \\
Lungs & $15(30)$ \\
Abdomen & $3(6)$ \\
Skin and soft tissues & $1(2)$ \\
Urinary tract & $5(10)$ \\
Other sites of infection & $6(12)$ \\
Positive blood cultures & \\
Pathogens & $17(34)$ \\
Enterobacteriaceae & $18(36)$ \\
Pseudomonas aeruginosa & $3(6)$ \\
Acinetobacter baumannii & \\
Antimicrobial treatment & $7(14)$ \\
Ceftazidime or cefepime & $16(32)$ \\
Piperacillin-tazobactam & $32(64)$ \\
Meropenem &
\end{tabular}

Data are presented as count (percentage).

effect resulted in higher drug concentrations in the LT compared to the ET phase (Table 3); TZP concentrations were significantly higher in the LT compared to the ET phase, despite similar PK and CRRT parameters. Median concentrations and PK parameters were similar for MEM in the two treatment phases.

Target concentrations were reached in 67/73 (92\%) of the TDMs (Figure 1); specifically, 8/9 TDMs for CEF (89\%), 43/44 (98\%) for MEM and 16/20 (80\%) for TZP. The proportions of patients reaching target drug concentrations were similar in the ET and LT phases (Table 3 ). The $\% \mathrm{~T}>4 \times$ tMIC was highly variable among patients (Figure 2) and drug concentrations were excessive in $39(53 \%)$ of the TDMs, $18 / 35(51 \%)$ in the ET and $22 / 38(58 \%)$ in the LT phase (Table 3). Calculating the proportion of TDMs with insufficient $\% \mathrm{~T}>4 \times \mathrm{MIC}$ for several MICs, we found no insufficient drug concentrations for CEF with MIC of $2 \mathrm{mg} / \mathrm{L}$ or less, for TZP with MIC of $8 \mathrm{mg} / \mathrm{L}$ or less and MEM for MIC of $1 \mathrm{mg} / \mathrm{L}$ or less (Table 4).

The $\mathrm{T}>4 \times$ tMIC was inversely correlated with the CRRT intensity $(r=-0.24, P=0.03)$ (Figure 3 ). $\beta$-lactam antibiotics CL was also significantly correlated with the CRRT intensity $(r=0.32, P=0.007)$ (Figure 4$)$. Similarly, drug CL was significantly greater in the upper quartile levels of CRRT intensity whereas $\mathrm{T}>4 \times \mathrm{tMIC}$ was significantly lower (Figure 5 and 6). There were no other correlations between PK/PD parameters and clinical, biologic, laboratory or therapeutic variables (including APACHE II score on admission, SOFA score, lactate and albumin levels, arterial partial pressure of oxygen/inspired oxygen fraction $\left(\mathrm{PaO}_{2} / \mathrm{FiO}_{2}\right)$, mechanical ventilation and vasopressor therapy on the day of TDM).

\section{Discussion}

In this study, we showed that, during CRRT, use of $\beta$ lactam antibiotics at doses similar to those used in patients without AKI resulted in drug concentrations above the minimal target threshold in more than $90 \%$ of patients, both in the ET and LT phases of therapy. However, there was wide variability in drug concentrations over time, with very high levels in some patients. Significant, although weak, correlations were found between CRRT intensity and $\mathrm{T}>4 \times \mathrm{tMIC}$ and total drug CL; hence, CRRT intensity should be considered when determining dosage strategy in these patients.

During treatment with CRRT, the prediction of $\beta$-lactam antibiotics concentrations is challenging, as both $\mathrm{Vd}$ and total drug CL may be affected by the type of membrane, the mechanism of epuration (diffusion versus convection), the total delivered dose and the CRRT intensity [21]. Previous studies that have evaluated $\beta$-lactam antibiotics concentrations during CRRT have reported conflicting results. In two studies, an MEM regimen of 0.5 to $1.0 \mathrm{~g}$ $\mathrm{q} 12 \mathrm{~h}$ resulted in adequate drug concentrations to treat the GNB that were identified [22,23]. However, for TZP, doses of $4.0 \mathrm{~g}$ q12h resulted in insufficient drug levels to treat Enterobacteriaceae and P. aeruginosa [24], and increasing the drug dose to $4 \mathrm{~g}$ q8h maintained TZP concentrations largely above the target threshold of efficacy [17]. Finally, Malone et al. [18] showed that although a cefepime regimen of $2 \mathrm{~g}$ daily was sufficient to cover susceptible GNB, higher doses of up to $4 \mathrm{~g}$ /day were necessary for bacteria with MICs of at least $8 \mathrm{mg} / \mathrm{L}$. Similarly, Matzke et al. proposed that usual doses of ceftazidime be administered to maintain drug levels above the target threshold for pathogens with high MICs [11].

On the basis of these findings, recent recommendations propose daily doses of $1 \mathrm{~g}$ q12h for MEM, $2 \mathrm{~g}$ $\mathrm{q} 12 \mathrm{~h}$ for CEF and $4 \mathrm{~g}$ q8h for TZP [10], with minor alterations if CVVH or CVVHDF techniques are used. However, these studies were limited by small patientcohorts, by the use of different CRRT devices and techniques, by the analysis of stable predefined CRRT settings and by the evaluation of various MIC targets. Hence, these data are difficult to generalize to larger ICU populations, may not be relevant if CRRT settings are modified over time, which is common practice, and may not provide an adequate daily dose for the treatment of less susceptible GNB. Indeed, Seyler et al. showed that these recommended doses resulted in adequate $\beta$-lactam antibiotics concentrations only for pathogens with low MICs and that increased drug doses should be considered in the treatment of less susceptible strains [15]. However, although such an approach may limit under-dosing if $P$. aeruginosa or 
Table 3 Pharmacokinetic and pharmacodynamic data and characteristics of continuous renal replacement therapy (CRRT)

\begin{tabular}{|c|c|c|c|c|c|c|}
\hline \multirow[t]{2}{*}{ Time from start of antibiotic to sampling, days } & \multicolumn{3}{|c|}{ Early TDM $(n=35) 2(1-2]$} & \multicolumn{3}{|c|}{ Late TDM $(n=38) 5(3-14]$} \\
\hline & $\operatorname{CEF}(n=3)$ & MEM $(n=25)$ & $\operatorname{TZP}(n=7)$ & CEF $(n=6)$ & MEM $(n=19)$ & $\operatorname{TZP}(n=13)$ \\
\hline Volume of distribution, $\mathrm{L}$ & $19.4(15.8$ to 23.1$)$ & $33.3(9.2$ to 86.5$)$ & $18.5(11.5$ to 61.3$)$ & $19.2(11.4$ to 32.9$)$ & $28.8(17.26$ to 83.78$)$ & $18.2(7.94$ to 52.80$)$ \\
\hline Volume of distribution, $\mathrm{L} / \mathrm{kg}$ & $0.26(0.24$ to 0.28$)$ & $0.39(0.11$ to 1.20$)$ & 0.24 (0.17 to 0.87$)$ & 0.21 (0.16 to 0.51$)$ & $0.37(0.23$ to 1.18$)$ & $0.22(0.11$ to 0.76$)$ \\
\hline$t_{1 / 2}, h$ & 4.3 (1.5 to 9.7$)$ & $3.9(1.8$ to 22.8$)$ & $3.0(1.5$ to 61.3$)$ & $8.0(6.0 \text { to } 35.3)^{*}$ & $3.6(1.8$ to 10.7$)$ & 4.8 (1.8 to 7.8$)$ \\
\hline Drug clearance, $\mathrm{mL} / \mathrm{h}$ & $52.4(27.3$ to 120.9$)$ & 72.3 (21.4 to 283.0$)$ & $54.0(47.3$ to 185.6$)$ & $23.3(5.4 \text { to } 58.5)^{*}$ & $98.3(21.6$ to 174.1$)$ & 51.0 (16.1 to 140.83$)$ \\
\hline Concentration at $\mathrm{TO}, \mathrm{mg} / \mathrm{L}$ & $28.2(26.1$ to 49.0$)$ & 7.7 (2.0 to 32.2$)$ & $63.2(15.3$ to 115.2$)$ & $64.3(3.2 \text { to } 139.0)^{*}$ & $6.8(2.7$ to 23.2$)$ & 80.0 (19.5 to 243.0) \\
\hline Concentration at $\mathrm{T} 2, \mathrm{mg} / \mathrm{L}$ & 74.5 (52.0 to 75.0$)$ & 21.6 (7.8 to 80.2$)$ & 89.5 (55.0 to 211.0) & 93.4 (50.5 to 165.0$)$ & 22.1 (12.9 to 37.0$)$ & 168.4 (49.0 to 395.0$)$ \\
\hline Concentration at target, $\mathrm{mg} / \mathrm{L}$ & 41.6 (34.3 to 58.2$)$ & $18.6(6.1$ to 41.0$)$ & 82.0 (43.0 to 174.8$)$ & $79.3(9.6 \text { to } 202.0)^{*}$ & 16.1 (9.6 to 30.5$)$ & $140.0(42.0 \text { to } 349.0)^{*}$ \\
\hline $\mathrm{T}>4 \times \mathrm{tMIC}, \mathrm{h}$ & $7.2(6.2$ to 14.0$)$ & $7.0(4.5$ to 11.8$)$ & 6.3 (1.0 to 7.7$)$ & $15.5(6.8$ to 55.8$)$ & $7.1(6.0$ to 11.3$)$ & $7.7(6.1$ to 12.4$)$ \\
\hline Adequate concentrations & $3(100 \%)$ & $9(36 \%)$ & $1(14.3 \%)$ & $1(16.7 \%)$ & $9(47.4 \%)$ & $3(23.1 \%)$ \\
\hline Excessive concentrations & $0(0 \%)$ & $15(60 \%)$ & $3(42.9 \%)$ & $4(66.7 \%)$ & $10(52.6 \%)$ & $9(69.2 \%)$ \\
\hline SOFA score & $11(4$ to 14$)$ & $14(4$ to 19$)$ & $12(5$ to 16$)$ & 12 (9 to 13$)$ & 12 (5 to 20$)$ & 13 (9 to 22$)$ \\
\hline CRRT blood flow, mL/minute & $140(130$ to 150$)$ & 150 (130 to 200$)$ & 150 (140 to 180$)$ & $140(100$ to 200$)$ & 150 (120 to 300$)$ & $150(100$ to 180$)$ \\
\hline Dialysate, $\mathrm{mL} / \mathrm{h}$ & $0(0$ to 1,500$)$ & $1,500$ (0 to 2,000$)$ & $1,500$ (0 to 2,000$)$ & $0(0$ to 1,500$)$ & $1,000$ (0 to 2,000$)$ & $0(0$ to 2,000$)$ \\
\hline Ultrafiltrate, mL/h & $1,000(1,000$ to 1,750$)$ & $2,000$ (100 to 2,000$)$ & $1,500(1,500$ to 2,000$)$ & $1,500(1,000$ to 3,000$)$ & $1,500$ (100 to 2,000$)$ & $2,000(1,500$ to 2,000$)$ \\
\hline CRRT intensity, mL/kg/h & 16.6 (10.5 to 47.7$)$ & 36.14 (8.9 to 57.14 ) & 31.5 (15.7 to 57.5 ) & 24.0 (16.6 to 50$)$ & 34.2 (16.6 to 85.1$)$ & 28.6 (21.4 to 61.5$)$ \\
\hline
\end{tabular}




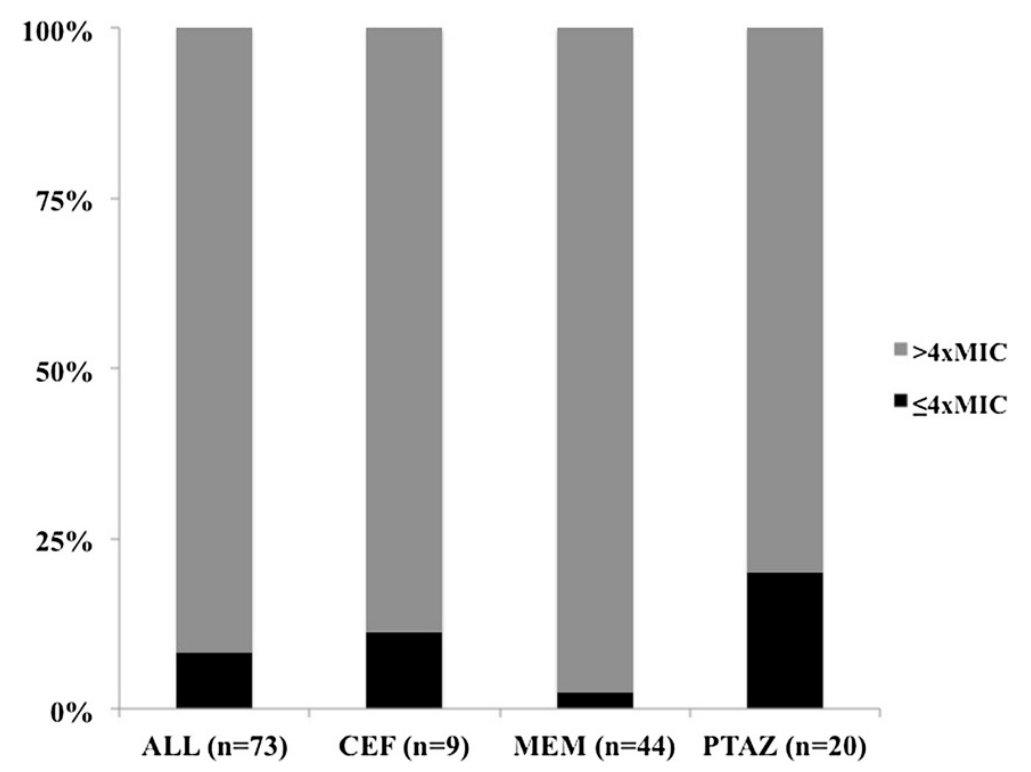

Figure 1 Proportion of patients with drug concentrations below or above four times the minimal inhibitory concentration (MIC) of Pseudomonas aeruginosa, for the different antibiotics. CEF, cephalosporins; MEM, meropenem; TZP, piperacillin/tazobactam.

other less susceptible strains are implicated, for more susceptible pathogens, drug regimens may need to be reduced. Moreover, more than $50 \%$ of samples in our study revealed very high drug concentrations in both the ET and LT phases. Drug accumulation and excessive $\beta$-lactam antibiotic concentrations may lead to adverse events, including neurological toxicity. Hallucinations, confusion, and seizures have been reported as a conse- quence of high $\beta$-lactam antibiotic concentrations, mostly in patients with renal impairment $[25,26]$, but also in patients with normal kidney function [27]. The mechanism of cerebral toxicity seems to be related to the drug interaction with the GABA-A receptor and is concentration-dependent [28]. Smith et al. reported a case of cefepime-related seizures during CRRT in a septic shock patient with a plasma concentration of $73.8 \mathrm{mg} / \mathrm{L}$,

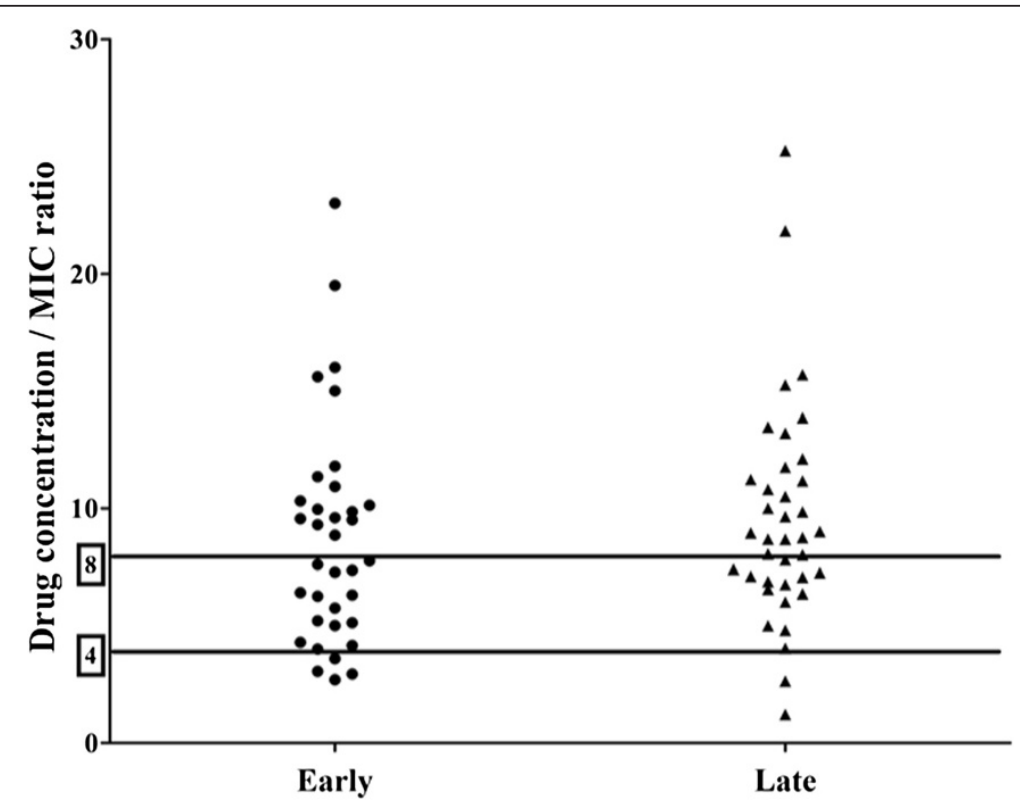

Figure 2 Distribution of the ratio between drug concentrations and the minimal inhibitory concentration (C/MIC) of Pseudomonas aeruginosa. Drug concentrations were considered at 40\%,50\% and 70\% for meropenem, piperacillin/tazobactam, and cephalosporin, respectively, and separated according to the early or late phase of therapy. Solid lines indicate a C/MIC of 4 and 8. 
Table 4 Proportion of therapeutic drug monitoring (TDMs) with insufficient $\%$ time $(T)>4 \times$ the minimal inhibitory concentration (MIC) for various MICs

\begin{tabular}{ccccc}
\hline \multirow{2}{*}{$\begin{array}{c}\text { MIC } \\
(\mathbf{m g} / \mathbf{L})\end{array}$} & $\begin{array}{c}\text { Target } \\
\text { concentrations (mg/L) }\end{array}$ & $\begin{array}{c}\text { Insufficient drug concentrations } \\
(\mathbf{n}=\mathbf{9})\end{array}$ & $\begin{array}{c}\text { TZP } \\
(\mathbf{n}=\mathbf{2 0})\end{array}$ & $\begin{array}{c}\text { MEM } \\
(\mathbf{n}=\mathbf{4 4})\end{array}$ \\
\hline $\mathbf{3 2}$ & $\mathbf{1 2 8}$ & $8(89)$ & $9(45)$ & $44(100)$ \\
$\mathbf{1 6}$ & $\mathbf{6 4}$ & $1(11)$ & $\mathbf{4}(\mathbf{2 0})$ & $44(100)$ \\
$\mathbf{8}$ & $\mathbf{3 2}$ & $\mathbf{1}(\mathbf{1 1 )}$ & 0 & $41(93)$ \\
$\mathbf{4}$ & $\mathbf{1 6}$ & $1(11)$ & 0 & $20(45)$ \\
$\mathbf{2}$ & $\mathbf{8}$ & 0 & 0 & $\mathbf{1}(\mathbf{2})$ \\
$\mathbf{1}$ & $\mathbf{4}$ & 0 & 0 & 0 \\
$\mathbf{0 . 5}$ & $\mathbf{2}$ & 0 & 0 & 0 \\
\hline
\end{tabular}

Data are expressed as counts (percentage). Boldface values indicate MIC corresponding to European Committee on Antimicrobial Susceptibility Testing (EUCAST) clinical breakpoints for Pseudomonas aeruginosa. CEF, cephalosporins; TZP, piperacillin/tazobactam; MEM, meropenem.

but cerebrospinal fluid levels of $6.1 \mathrm{mg} / \mathrm{L}$; seizures resolved after drug discontinuation [29]. Chapuis et al. described two patients who developed seizures with trough cefepime concentrations $>20 \mathrm{mg} / \mathrm{L}$ [30]. We did not collect neurological data in our patients so we cannot relate drug levels to neurological complications.

With the high PK variability among patients, an important finding of our study is the correlation between CRRT intensity and drug CL. In a recent study, Roberts et al. found no association between broad-spectrum $\beta$ lactam antibiotics and PK and CRRT flow rate [14]. However, the sample size was very limited and drug regimens differed among patients. In other studies, the total CL of TZP, MEM and CEF were strongly correlated to dialysate and ultrafiltration rates [11,13,24]. In a study in six patients, Valtonen et al. showed that MEM CL was significantly lower during a flow rate of $1 \mathrm{~L} / \mathrm{h}$ using CVVH when compared to $2 \mathrm{~L} / \mathrm{h}$ using CVVHDF [13]. In another study, the same authors showed that TZP CL increased from $3.89 \pm 1.23 \mathrm{~L} / \mathrm{h}$ to $5.48 \pm 2.11 \mathrm{~L} / \mathrm{h}$ when CRRT intensity was doubled [24]. More recently, Covajes et al. reported that high CRRT intensity was independently associated with lower vancomycin levels on day 1 of therapy [31]. Although other factors, such as filter age, membrane absorption and residual renal function are all potential confounders when trying to determine a clear relationship between $\beta$-lactam antibiotic elimination and CRRT intensity, this parameter should be taken into account and prospectively studied when evaluating optimal dosing strategy during CRRT.

Our study has some limitations. First, $\beta$-lactam antibiotics concentrations were sampled at different time points after the onset of therapy and this may not always represent a steady state. Second, because of the retrospective nature of the study, it was not possible to correct drug prescription for delay and interruption of CRRT during therapy, which may have altered drug CL. In addition, unbound free drug concentrations are a major determinant of the total antibiotic CL. Drug binding to circulating proteins, such as albumin, contributes to the decrease in the passage of TZP across CRRT membrane, although it cannot completely explain the findings obtained in several clinical studies [32]. Although we considered that protein binding was negligible for CEF and MEM, we did not measure free drug levels for TZP, which has an estimated protein binding of 25 to $30 \%$, and this may be a significant confounder in this setting. Third, our target concentrations

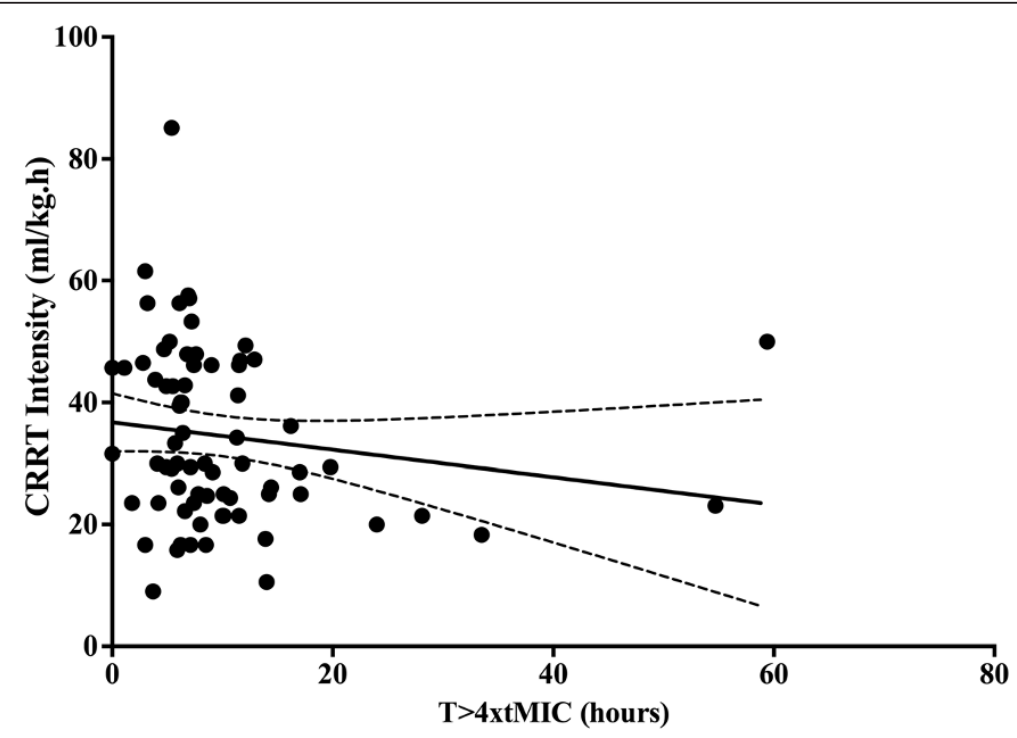

Figure 3 Correlation between continuous renal replacement therapy (CRRT) intensity and the time (h) above four times the minimal inhibitory concentration (MIC) of Pseudomonas aeruginosa. 


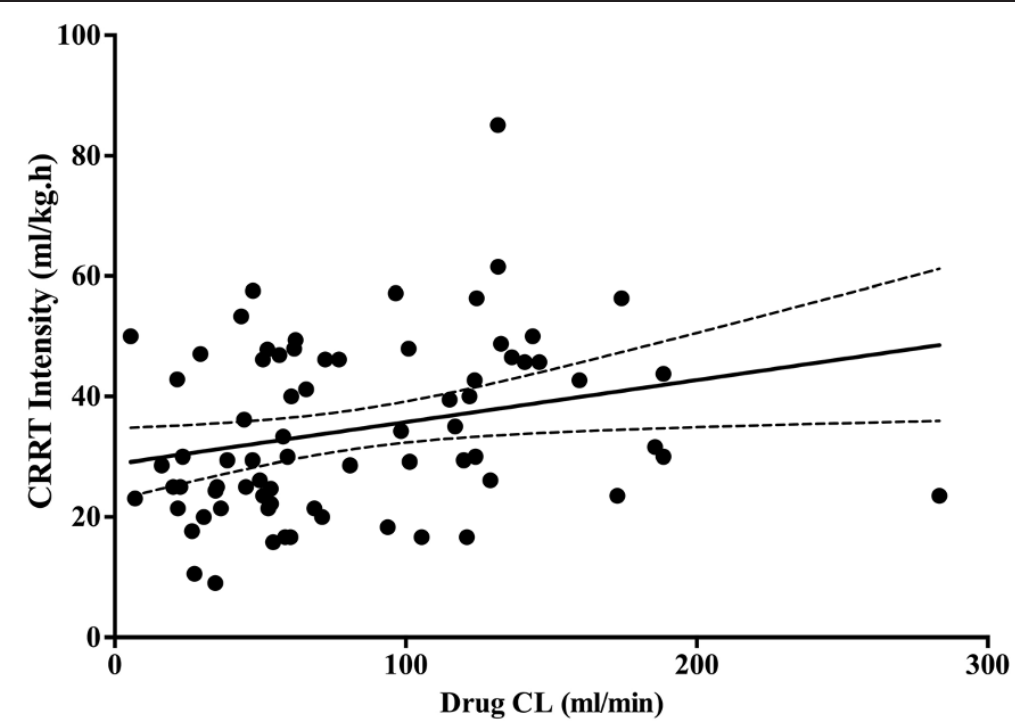

Figure 4 Correlation between continuous renal replacement therapy (CRRT) intensity and drug clearance (CL).

could be criticized because no clear threshold of effectiveness has been defined for $\beta$-lactam antibiotics in the treatment of life-threatening infections, and $\mathrm{T}>\mathrm{MIC}$ and $\mathrm{T}>4 \times$ MIC have both been shown to be associated with a better clinical response [33,34]. Significant variation observed in the PK targets supports the need for further studies to define optimal drug concentrations for ICU patients [35]. Also, we did not specifically measure MICs of isolated bacteria and significant differences in final results would have been obtained if target MICs other than those of $P$. aeruginosa were considered. Indeed, when the isolated bacteria are found to be susceptible and have a low MIC (as for some Enterobacteriaceae), it is hard to justify maintaining the same therapeutic targets as for Pseudomonas, and exposing the patients to high concentrations and to potential toxicity. Accordingly, dose adjustment should probably be considered along with drug de-escalation, not only according to TDM but also to bacteria susceptibility and MIC $[36,37]$. Fourth, no data on efficacy that is, clinical or microbiological responses) were collected so that no conclusion on the role of routine measurements of $\beta$-lactam antibiotics concentration can be drawn from our results. Fifth, although we compared drug concentrations in the early and late phase of therapy, we

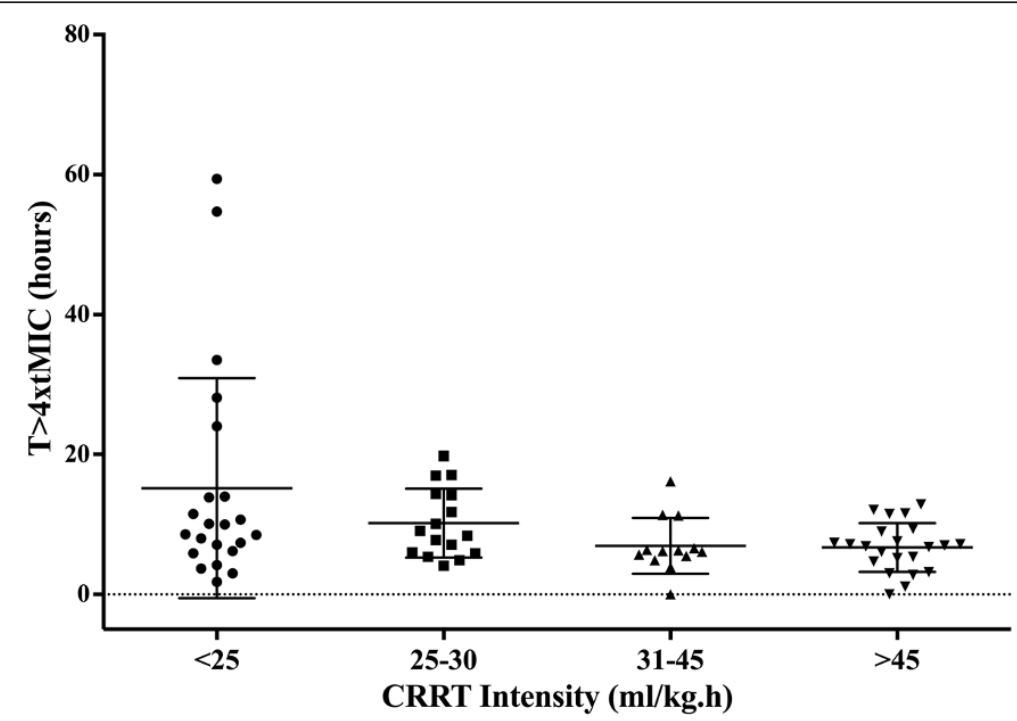

Figure 5 The time (T) above four times the target $(\mathrm{t})$ minimal inhibitory concentration (MIC) $(\mathrm{T}>\times$ of Pseudomonas aeruginosa was lower in patients with higher continuous renal replacement therapy (CRRT) intensity $(<25 \mathrm{~mL} / \mathrm{kg} . \mathrm{h}=9.3$ (ranges $=1.8$ to 59.4$) \mathrm{h}$; 25 to $30 \mathrm{~mL} / \mathrm{kg} \cdot \mathrm{h}=8.7$ (4.1 to 19.8$) \mathrm{h} ; 31$ to $45 \mathrm{~mL} / \mathrm{kg} \cdot \mathrm{h}=6.2$ (0 to 16.2$) \mathrm{h} ;>45 \mathrm{~mL} / \mathrm{kg} \cdot \mathrm{h}=6.9$ (0 to 12.9$) \mathrm{h} ; P=0.01$ ). 


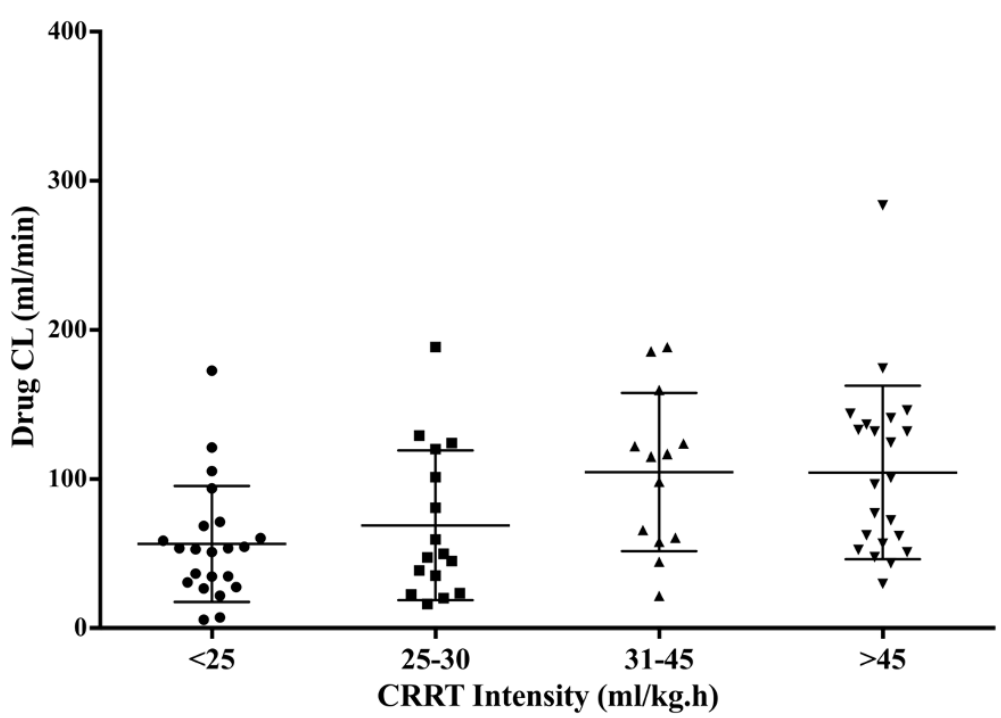

Figure 6 Drug clearance (CL) was greater in patients with higher continuous renal replacement therapy (CRRT) intensity $(<25 \mathrm{~mL} / \mathrm{kg} . \mathrm{h}=53$ (ranges $=5$ to 172 ) mL/minute; 25 to $30 \mathrm{~mL} / \mathrm{kg} . \mathrm{h}=49$ (16 to 188 ) mL/minute; 31 to $45 \mathrm{~mL} / \mathrm{kg} . \mathrm{h}=115$ (21 to 188 ) $\mathrm{mL} / \mathrm{minute} ;>45 \mathrm{~mL} / \mathrm{kg} . \mathrm{h}=99$ (30 to 283 ) $\mathrm{mL} /$ minute; $P=0.02$ ).

did not assess the two time points for every patient and this latter approach would have probably been more informative in this setting. Finally, we did not collect the number of patients treated with CRRT and receiving these drugs but whose $\beta$-lactam concentrations were not assessed; this may be an important selection bias with only the most severe patients who were included, which may lead to an overestimation of the problem.

\section{Conclusions}

During CRRT, $\beta$-lactam antibiotics regimens similar to those recommended for patients with normal renal function should be given to avoid under-dosing as empirical therapy. However, drug accumulation occurs rapidly and daily doses should be rapidly reduced, especially in case of very susceptible bacteria. Given the wide variability in drug PK parameters in this population of patients, TDM could be considered to adjust drug regimens. Drug prescription should also take into account the intensity of CRRT.

\section{Key messages}

- The use of standard doses of $\beta$-lactam antibiotics during CRRT may result in inadequate serum concentrations to treat less susceptible Gram-negative strains, such as P. aeruginosa.

- During CRRT, the administration of drug regimens similar to those patients with normal renal function reduced the proportion of inadequate serum concentrations to $10 \%$.
- Nevertheless, $53 \%$ of samples were associated with very high drug levels and daily drug regimens may need to be adapted accordingly to avoid adverse events.

\section{Abbreviations}

AKI: acute kidney injury; APACHE: acute physiology and chronic health evaluation; CEFP: ceftazidime or cefepime; $\mathrm{CL}$ : clearance; $\mathrm{CrCl}$ : creatinine clearance; CRRT: continuous renal replacement therapy; CWHDF: continuous veno-venous hemodiafiltration; CWHF: continuous veno-venous hemofiltration; ECMO: extracorporeal membrane oxygenation; ET: early phase; EUCAST: European Committee on Antimicrobial Susceptibility Testing; GNB: Gram-negative bacteria; LT: late phase; MEM: meropenem; MIC: minimal inhibitory concentration; $\mathrm{PaO}_{2} / \mathrm{FiO}_{2}$ : arterial partial pressure of oxygen/inspired oxygen fraction; PK: pharmacokinetics; SOFA: sequential organ failure assessment; TDM: therapeutic drug monitoring; TZP: piperaacillin/ tazobactam; Vd: volume of distribution.

\section{Competing interests}

The author declared that they have no competing interests.

\section{Author contributions}

MB, FJ and FST contributed to the conception and design of the study protocol. GSC, LS, JLV and MH participated in the coordination of the study and data collection. FC performed the pharmacokinetics analyses and contributed to the analysis and interpretation of the data. MB, GCS, FJ, JLV and FST drafted the manuscript. All authors were involved in revising it critically for important intellectual content. All authors read and gave final approval of the present version of the manuscript to be published.

\section{Acknowledgements}

The authors would like to thank the ICU staff for their support. The study was not supported by any funding.

\section{Author details}

'Department of Intensive Care, Erasme Hospital, Université Libre de Bruxelles (ULB), Route de Lennik, 808, 1070 Brussels, Belgium. ${ }^{2}$ Department of Infectious Diseases, Erasme Hospital, Université Libre de Bruxelles (ULB), Route de Lennik, 808, 1070 Brussels, Belgium. ${ }^{3}$ Department of Clinical Biochemistry, Erasme Hospital, Université Libre de Bruxelles (ULB), Route de Lennik, 808, 1070 Brussels, Belgium. 
Received: 10 January 2014 Accepted: 7 May 2014

Published: 22 May 2014

\section{References}

1. Vincent JL, Rello J, Marshall J, Silva E, Anzueto A, Martin CD, Moreno R, Lipman J, Gomersall C, Sakr Y, Reinhart K, EPIC II, Group of Investigators: International study of the prevalence and outcomes of infection in intensive care units. JAMA 2009, 302:2323-2329.

2. Brun-Buisson C: The epidemiology of the systemic inflammatory response. Intensive Care Med 2000, 26:S64-S74.

3. Kumar A, Roberts D, Wood KE, Light B, Parrillo JE, Sharma S, Suppes R, Feinstein D, Zanotti S, Taiberg L, Gurka D, Kumar A, Cheang M: Duration of hypotension before initiation of effective antimicrobial therapy is the critical determinant of survival in human septic shock. Crit Care Med 2006, 34:1589-1596.

4. Dellinger RP, Levy MM, Rhodes A, Annane D, Gerlach H, Opal SM, Sevransky JE, Sprung CL, Douglas IS, Jaeschke R, Osborn TM, Nunnally ME, Townsend SR Reinhart K, Kleinpell RM, Angus DC, Deutschman CS, Machado FR, Rubenfeld GD, Webb S, Beale RJ, Vincent JL, Moreno R, Surviving Sepsis Campaign Guidelines Committee including The Pediatric Subgroup: Surviving Sepsis Campaign: international guidelines for management of severe sepsis and septic shock, 2012. Intensive Care Med 2013, 39:165-228.

5. Kollef MH, Sherman G, Ward S, Fraser VJ: Inadequate antimicrobial treatment of infections: a risk factor for hospital mortality among critically ill patients. Chest 1999, 115:462-474.

6. Zaragoza R, Artero A, Camarena JJ, Sancho S, González R, Nogueira JM: The influence of inadequate empirical antimicrobial treatment on patients with bloodstream infections in an intensive care unit. Clin Microbiol Infect 2003, 9:412-418

7. Pinder M, Bellomo R, Lipman J: Pharmacological principles of antibiotic prescription in the critically ill. Anaesth Intensive Care 2002, 30:134-144.

8. Adnan S, Li JX, Wallis SC, Rudd M, Jarrett P, Paterson DL, Lipman J, Udy AA, Roberts JA: Pharmacokinetics of meropenem and piperacillin in critically ill patients with indwelling surgical drains. Int J Antimicrob Agents 2013, 42:90-93.

9. Heinemeyer G, Link J, Weber W, Meschede V, Roots I: Clearance of ceftriaxone in critical care patients with acute renal failure. Intensive Care Med 1990, 16:448-453.

10. Trotman RL, Williamson JC, Shoemaker DM, Salzer WL: Antibiotic dosing in critically ill adult patients receiving continuous renal replacement therapy. Clin Infect Dis 2005, 41:1159-1166.

11. Matzke GR, Frye RF, Joy MS, Palevsky PM: Determinants of ceftazidime clearance by continuous venovenous hemofiltration and continuous venovenous hemodialysis. Antimicrob Agents Chemother 2000, 44:1639-1644.

12. Mueller SC, Majcher-Peszynska J, Hickstein H, Francke A, Pertschy A, Schulz M, Mundkowski R, Drewelow B: Pharmacokinetics of piperacillin-tazobactam in anuric intensive care patients during continuous venovenous hemodialysis. Antimicrob Agents Chemother 2002, 46:1557-1560.

13. Valtonen M, Tiula E, Backman JT, Neuvonen PJ: Elimination of meropenem during continuous veno-venous haemofiltration and haemodiafiltration in patients with acute renal failure. J Antimicrob Chemother 2000, 45:701-704.

14. Roberts DM, Roberts JA, Roberts MS, Liu X, Nair P, Cole L, Lipman J, Bellomo R, RENAL Replacement Therapy Study Investigators: Variability of antibiotic concentrations in critically ill patients receiving continuous renal replacement therapy: a multicentre pharmacokinetic study. Crit Care Med 2012, 40:1523-1528.

15. Seyler L, Cotton F, Taccone FS, De Backer D, Macours P, Vincent JL, Jacobs F: Recommended $\beta$-lactam regimens are inadequate in septic patients treated with continuous renal replacement therapy. Crit Care 2011, 15:R137.

16. Levy MM, Fink MP, Marshall JC, Abraham E, Angus D, Cook D, Cohen J, Opal SM, Vincent JL, Ramsay G: International Sepsis Definitions Conference. Intensive Care Med 2003, 29:530-538.

17. van der Werf TS, Mulder PO, Zijlstra JG, Uges DR, Stegeman CA: Pharmacokinetics of piperacillin and tazobactam in critically ill patients with renal failure, treated with continuous veno-venous hemofiltration (CVVH). Intensive Care Med 1997, 23:873-877.

18. Malone RS, Fish DN, Abraham E, Teitelbaum I: Pharmacokinetics of cefepime during continuous renal replacement therapy in critically ill patients. Antimicrob Agents Chemother 2001, 45:3148-3155.
19. Wolff F, Deprez G, Seyler L, Taccone F, Hites M, Gulbis B, Vincent JL, Jacobs F, Cotton F: Rapid quantification of six $\beta$-lactams to optimize dosage regimens in severely septic patients. Talanta 2013, 103:153-160.

20. Clinical Breakpoint for Gram Negative Bacteria. [http://www.eucast.org/ fileadmin/src/media/PDFs/EUCAST_files/ Breakpoint_tables/Breakpoint_ table_v_3.1.pdf] (Last accessed 14 October 2013).

21. Choi G, Gomersall CD, Tian Q, Joynt GM, Freebairn R, Lipman J: Principles of antibacterial dosing in continuous renal replacement therapy. Crit Care Med 2009, 37:2268-2282.

22. Ververs TF, van Dijk A, Vinks SA, Blankestijn PJ, Savelkoul JF, Meulenbelt J, Boereboom FT: Pharmacokinetics and dosing regimen of meropenem in critically ill patients receiving continuous venovenous hemofiltration. Crit Care Med 2000, 28:3412-3416.

23. Krueger WA, Schroeder TH, Hutchison M, Hoffmann E, Dieterich HJ, Heininger A, Erley C, Wehrle A, Unertl K: Pharmacokinetics of meropenem in critically ill patients with acute renal failure treated by continuous hemodiafiltration. Antimicrob Agents Chemother 1998, 42:2421-2424.

24. Valtonen M, Tiula E, Takkunen O, Backman JT, Neuvonen PJ: Elimination of the piperacillin/tazobactam combination during continuous venovenous haemofiltration and haemodiafiltration in patients with acute renal failure. J Antimicrob Chemother 2001, 48:881-885.

25. Gangireddy VGR, Mitchell LC, Coleman T: Cefepime neurotoxicity despite renal adjusted dosing. Scand I Infect Dis 2011, 43:827-829.

26. Huang WT, Hsu YJ, Chu PL, Lin SH: Neurotoxicity associated with standard doses of piperacillin in an elderly patient with renal failure. Infection 2009, 37:374-376.

27. Maganti R, Jolin D, Rishi D, Biswas A: Nonconvulsive status epilepticus due to cefepime in a patient with normal renal function. Epilepsy Behav 2006 8:312-314.

28. Klion AD, Kallsen J, Cowl CT, Nauseef WM: Ceftazidime-related nonconvulsive status epilepticus. Arch Int Med 1994, 154:586-589.

29. Smith NL, Freebairn RC, Park MA, Wallis SC, Roberts JA, Lipman J: Therapeutic drug monitoring when using cefepime in continuous renal replacement therapy: seizures associated with cefepime. Critical Care Resusc 2012, 14:312-315.

30. Chapuis TM, Giannoni E, Majcherczyk PA, Chioléro R, Schaller MD, Berger MM, Bolay S, Décosterd LA, Bugnon D, Moreillon P: Prospective monitoring of cefepime in intensive care unit adult patients. Crit Care 2010, 14:R51.

31. Covajes C, Scolletta S, Penaccini L, Ocampos-Martinez E, Abdelhadii A, Beumier M, Jacobs F, de Backer D, Vincent IL, Taccone FS: Continuous infusion of vancomycin in septic patients receiving continuous renal replacement therapy. Intern J Antimicrob Agents 2013, 41:261-266.

32. Arzuaga A, Isla A, Gascón AR, Maynar J, Corral E, Pedraz JL: Elimination of piperacillin and tazobactam by renal replacement therapies with AN69 and polysulfone hemofilters: evaluation of the sieving coefficient. Blood Purif 2006, 24:347-354.

33. McKinnon PS, Paladino JA, Schentag JJ: Evaluation of area under the inhibitory curve (AUIC) and time above the minimum inhibitory concentration ( $>$ MIC) as predictors of outcome for cefepime and ceftazidime in serious bacterial infections. Int J Antimicrob Agents 2008, 31:345-351.

34. Tam VH, McKinnon PS, Akins RL, Rybak MJ, Drusano GL: Pharmacodynamics of cefepime in patients with Gram-negative infections. J Antimicrob Chemother 2002, 50:425-428.

35. Wong G, Brinkman A, Benefield RJ, Carlier M, De Waele JJ, El Helali N, Frey O, Harbarth S, Huttner A, McWhinney B, Misset B, Pea F, Preisenberger J, Roberts MS, Robertson TA, Roehr A, Sime FB, Taccone FS, Ungerer JP, Lipman J, Roberts $J A$ : An international, multicentre survey of $\beta$-lactam antibiotic therapeutic drug monitoring practice in intensive care units. $J$ Antimicrob Chemother 2014, Epub ahead of print

36. Nicasio AM, Eagye KJ, Kuti EL, Nicolau DP, Kuti JL: Length of stay and hospital costs associated with a pharmacodynamic-based clinical pathway for empiric antibiotic choice for ventilator-associated pneumonia. Pharmacotherapy 2010, 30:453-462.

37. Goncalves-Pereira J, Paiva JA: Dose modulation: a new concept of antibiotic therapy in the critically ill patient? J Crit Care 2013, 28:341-346.

doi:10.1186/cc13886

Cite this article as: Beumier et al:: $\beta$-lactam antibiotic concentrations during continuous renal replacement therapy. Critical Care 2014 18:R105. 